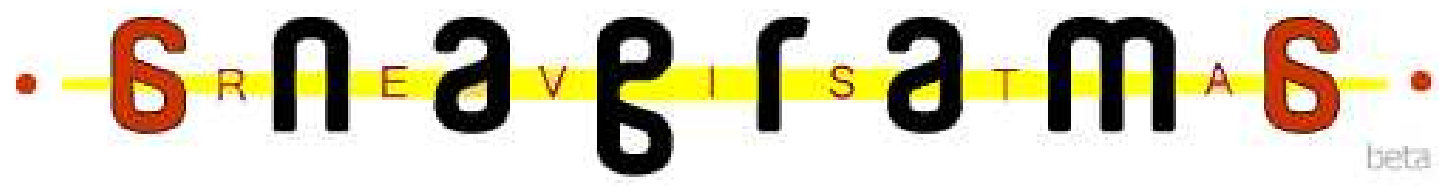

\section{Fandom: cultura participatiua em busca de um ídolo}

\section{Camila Monteiro ${ }^{7}$}

\section{Resumo}

O presente trabalho tem como objetivo estudar o funcionamento dos chamados fandoms na internet, em especial os do reality show norte-americano American Idol e como eles refletem a convergência das mídias, inteligência coletiva, troca de capital social e remediação. Para o desenvolvimento da pesquisa foram estudados dois diferentes fandoms: o Idolforums e o ONTD_AI. Ambos são fóruns norte-americanos, abertos ao público para a discussão de assuntos referentes ao programa. Como os objetos de estudo são dois fandoms da internet que retratam um programa de TV cujo objetivo é encontrar um ídolo musical, a convergência de mídias juntamente com a cultura participativa (Jenkins, 2008) são carros-chefe deste trabalho.

Palauras-chave: Fandom; Fórum; Convergência de Mídias; Remediação; Inteligência Coletiva.

\footnotetext{
${ }^{1}$ Acadêmica do curso de Comunicação Social - Habilitação Jornalismo da Universidade Católica de Pelotas.
} 


\section{Introdução}

Derivado do termo em latim fanaticus, que etimologicamente é "devoto, pertencente a um templo", o significado da palavra fã sempre foi relacionado a uma série de estereótipos. Nerds, apaixonados por Star Trek e games, sem vida social, obcecados pelos ídolos e muitas vezes psicóticos (habitualmente comparados a Mark David Chapman, fã que assassinou John Lennon), os fãs pertenciam ao underground, eram marginalizados tanto pela sociedade em geral, como pelas grandes mídias.

Atualmente, como observa Jenkins (2006) a realidade dos fandoms, os grupos de fãs, mudou, e eles não somente fazem parte do mainstream, como são considerados o centro das atenções dos grandes conglomerados midiáticos.

Construindo a sua própria cultura e identidade através da interação social (Recuero 2009) e da apropriação e remediação (Bolter e Gruisin, 2000) de meios de comunicação de massa, os fandoms são exemplos concretos da inteligência coletiva estudada por Lévy (1998), aonde "ninguém sabe tudo, todos sabem alguma coisa, todo o saber está na humanidade".

Esse trabalho procura mostrar como esses fenômenos, juntamente com a convergência das mídias, agem dentro de dois fandoms sobre o reality show norteamericano American Idol. Eles são: o fórum $\operatorname{IDF}^{2}$ (Idolforums) e a comunidade ONTD_AI ${ }^{3}$ (Oh no they didn't American Idol).

\section{A força do American idol}

Em meio a centenas de opções dadas pelos meios de comunicação, é cada vez mais complicado atingir altos índices de audiência, concentrando um grande público em um só programa. Vivemos a era das subculturas de nichos, definido por Anderson (2006: 182), como "um mundo de escolhas abundantes, pessoas que tem mais identificação, dividem os mesmos gostos".

Atingindo tanto as culturas de nicho, como a cultura de massa, o reality show norteamericano American Idol é há cerca de sete anos, o programa mais visto dos Estados Unidos. Com um nome auto-explicativo, o programa que passa de janeiro a maio, busca

\footnotetext{
${ }^{2}$ http://idolforums.com.

3 http://community.livejournal.com/ontd_ai.
} 
um ídolo musical. Apresentado pelo radialista Ryan Seacrest, e com três jurados fixos (Simon Cowell, Randy Jackson e Paula Abdul, substituída este ano por Ellen DeGeneres), o programa está em sua nona edição e tem uma média de audiência de 21 milhões de pessoas.

Ao longo destes sete anos de existência, o programa já revelou nomes fortes no mercado musical, como a cantora pop Kelly Clarkson, a banda de rock Daughtry, a atriz Jennifer Hudson e cantora country Carrie Underwood. Estima-se que juntando os oito vencedores do programa até hoje, a franquia já vendeu mais de 16 milhões de CDs, número bastante alto para o mercado fonográfico atual, que nos últimos anos vem sofrendo com o declínio na vendagem de álbuns.

Esses números conquistados, aumentam os investimentos do programa a cada ano que passa, considerando o fato de o American Idol não ser apenas um programa de televisão, mas uma franquia transmidiática, envolvendo também a indústria fonográfica, empresas telefônicas e a internet.

É possível entender esse fenômeno que é o reality show, através das técnicas da “economia afetiva” (Roberts, 2005), utilizadas cada vez mais pela indústria televisiva.

A economia afetiva é baseada na vontade dos grupos de fãs do programa, procurando compreender o consumo midiático no ponto de vista desses fãs, oferecendo aquilo que eles desejam para dessa forma, moldar as vontades do consumidor e direcionar as decisões de compra dos mesmos (Jenkins, 2008).

Desta forma, é estabelecida uma nova relação com os consumidores, as chamadas "lovemarks", que segundo Roberts (2005) são muito mais eficientes do que as marcas tradicionais, pois conquistam o amor e respeito dos consumidores. E é esse o objetivo do American Idol, fazer com que seus fãs tenham esse amor, sintam-se inseridos dentro do programa, participem, votem semanalmente, envolvam-se, criando uma fidelidade tanto com a franquia, como com os patrocinadores.

O número de pessoas que assiste a série é muito maior do que o das que fazem os testes; o número de candidatos que fazem teste é muito maior do que os que vão ao ar; o número daqueles que vão ao ar é muito maior do que os que se tornam finalistas. Mas, em cada passo ao longo do caminho, os espectadores são convidados a imaginar que "poderia ser eu, ou alguém que eu conheço". A partir daí, a votação semanal aumenta o envolvimento dos espectadores, construindo uma forte lealdade a determinados candidatos. Quando os discos são lançados, muitos dos consumidores já apoiaram os candidatos, e fãs-clubes já estão envolvidos em marketing alternativo (Jenkins, 2008 p.106) 
Adquirir a fidelidade do telespectador é essencial para a obtenção do sucesso, e como o American Idol dá ao telespectador a chance de participar da construção da carreira musical de alguém, a franquia possui milhares de seguidores, e muitos grupos de fãs espalhados na internet que são especializados no assunto.

\section{Interagindo em um fandom}

Assistir ao programa é apenas o começo, de um longo processo de participação dentro de um fandom. As conexões dentro desse grupo de fãs são construídas através dos laços sociais, que por sua vez são formados através da interação social entre os atores (no caso, usuários do fórum) (Recuero, 2009). Dentro de um fandom, a interação é essencial para um crescimento de qualidade do fórum. E dessa forma, graças a maior proximidade dos membros, os laços entre eles ficam cada vez mais fortes.

Em vez de nos relacionarmos por laços frouxos com multidões, graças às sobreposições da cultura de massa, temos a capacidade de nos interligarmos, mediante laços mais fortes, com igual quantidade de pessoas, se não com mais, em conseqüência da afinidade comum pela cultura de nicho. (Anderson, 2005 p. 189)

Os laços sociais em um fórum são na maioria das vezes dialógicos (Recuero, 2009) e dados através de interações mútuas (Primo, 2007), na forma de discussões nos diferentes tópicos existentes.

Lévy (1998: 32) afirma que esses laços sociais criados "suscitam coletivos inteligentes e valorizam ao máximo a diversidade das qualidades humanas”.

Os dois fandoms estudados possuem formas de interação similares. O primeiro, o Idolforums, foi criado em 2003 e tem aproximadamente 98 mil membros registrados. $\mathrm{O}$ ONTD_AI, é uma comunidade do livejournal ${ }^{4}$, foi criada no inicio de 2009 e tem 6.137 membros. No IDF, existem sub-fórums de esporte, outros reality shows, filmes, entretenimento em geral. Já na comunidade ONTD_AI, o assunto é exclusivamente American Idol.

\footnotetext{
${ }^{4}$ http://www.livejournal.com. Livejournal, também conhecido como LJ é uma comunidade virtual aonde os usuários podem criar blogs, jornais e diários.
} 

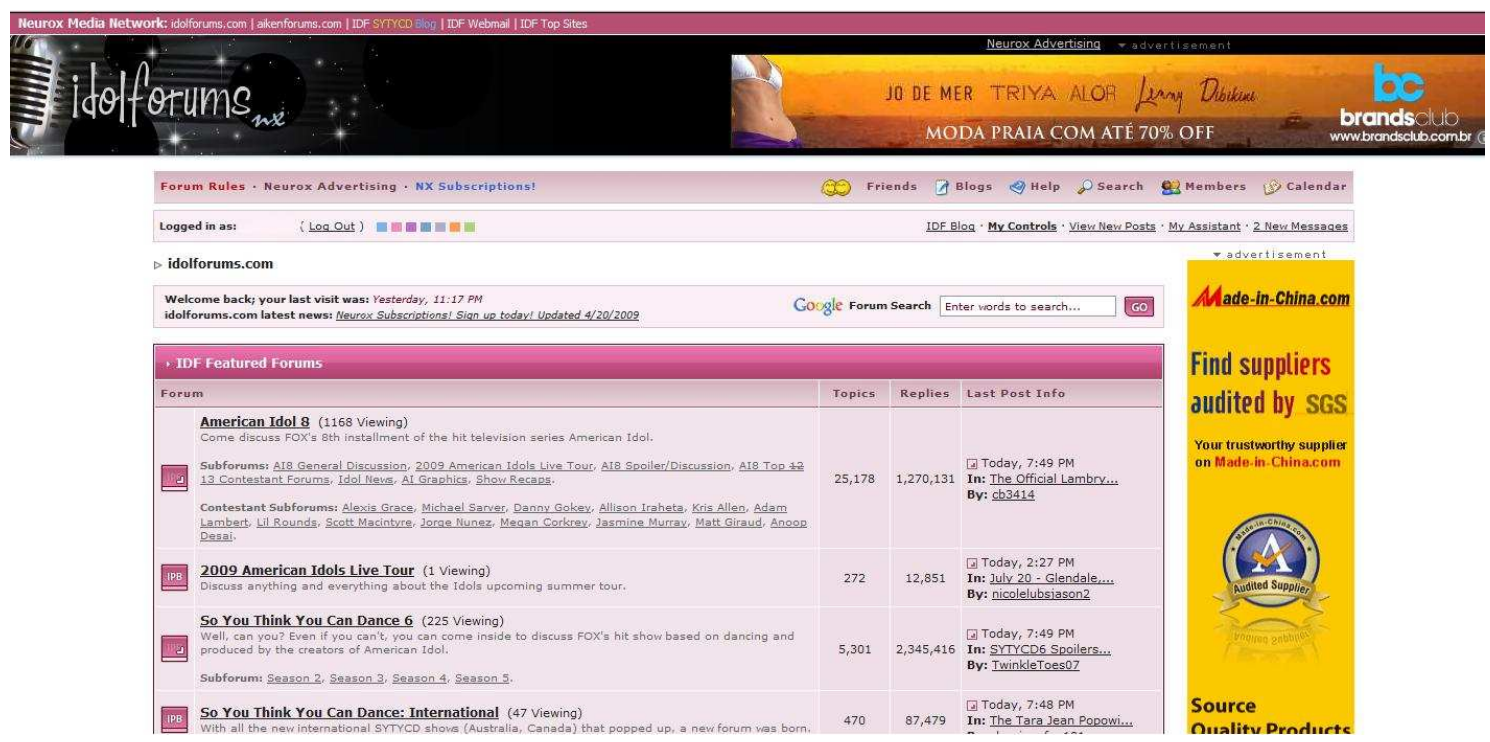

Fig 1: Página inicial do IDF, Idolforums

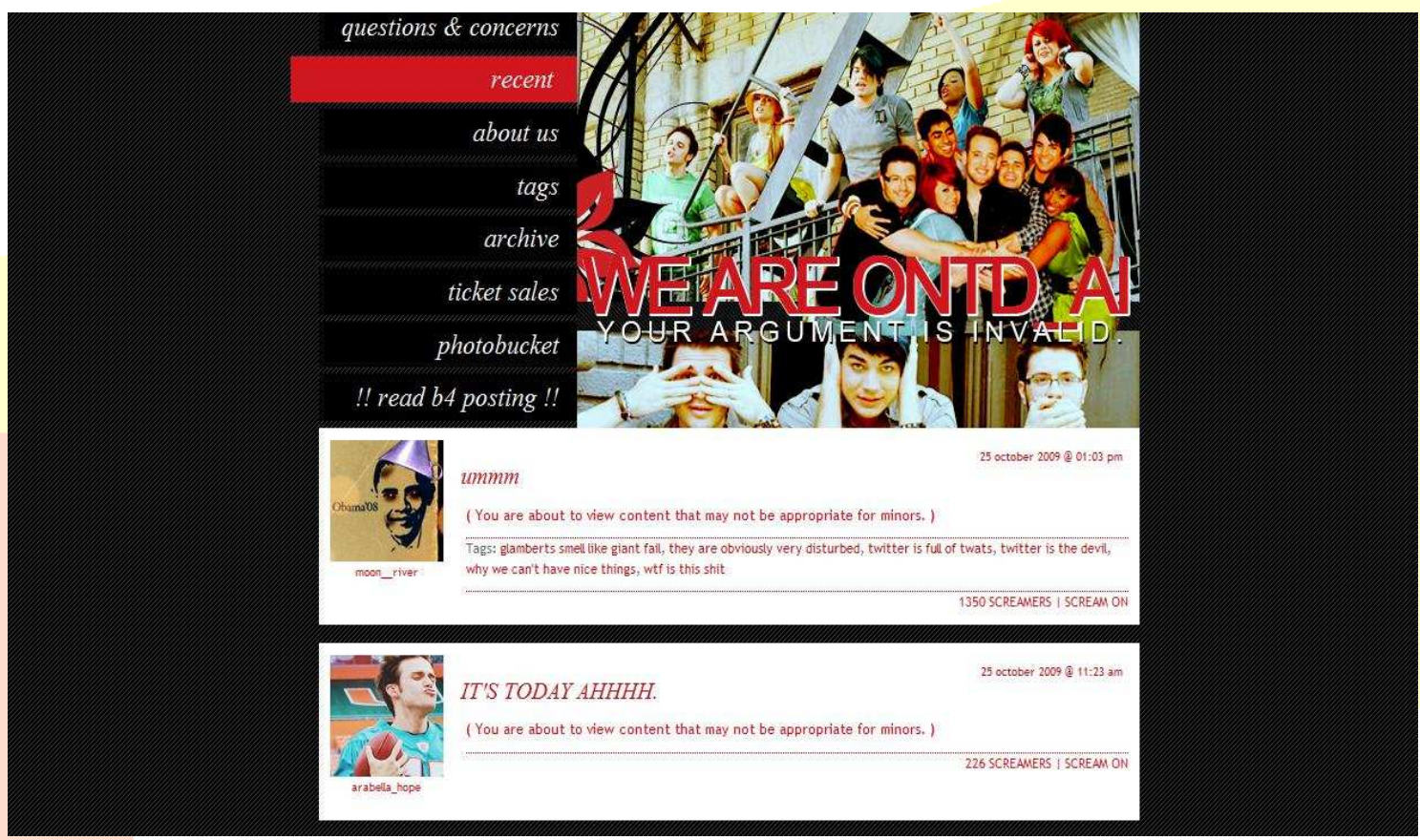

Fig 2: Página inicial do grupo ONTD_AI

Muitos conteúdos dentro desses fóruns são proibidos para convidados ou para os chamados "lurkers", pessoas que mesmo cadastradas, participam raramente das

\footnotetext{
${ }^{5}$ Termo utilizado nos fandoms em referência aos usuários que ficam "escondidos" na comunidade, sem nenhuma, ou pouca participação no fórum.
}

Reuista Anagrama: Reuista Científica Interdisciplinar da Graduação

\author{
Ano 4 - Edição 1 Setembro-nouembro de 2010
}

Auenida Professor Lúcio Martins Rodrigues, Ч४३, Cidade Uniuersitária, São Paulo, CEP: 05508-900 
discussões. Ser um membro ativo dentro destes grupos é essencial para ter acesso a todos os conteúdos publicados.

Para não perder novidades dentro de um fórum, é de extrema importância que ocorra troca de capital social entre os interagentes. Determinado pelos conteúdos das relações sociais, o capital social é "um conjunto de recursos de um determinado grupo, que pode ser usufruído por todos os membros do grupo, ainda que individualmente" (Recuero, 2009: 50), podendo ser relacional, normativo, cognitivo e institucional de confiança do ambiente social. Tanto no IDF como no ONTD_AI, a troca de capital é relacional, através da soma das relações, que conectam os usuários que ali se encontram.

\section{Fandoms inteligentes}

Um dos fatores mais interessantes dentro desses fóruns é o processo de aquisição de conhecimento a partir da interação com outros usuários.

E é dessa forma que é consolidada a inteligência coletiva. Lévy (1998: 28) afirma que "a inteligência coletiva é distribuída por toda parte, incessantemente valorizada, coordenada tem tempo real, e que resulta em uma mobilização efetiva das competências."

A inteligência coletiva é diretamente ligada à cultura participativa. Estamos cada vez mais dependentes dos outros, pois é impossível saber de tudo o tempo inteiro.

Nosso local de trabalho tornou-se mais cooperativo; nosso processo político tornou-se mais descentralizado; estamos vivendo cada vez mais no interior de culturas baseadas na inteligência coletiva. Nossas escolas não estão mais ensinando o que significa viver e trabalhar em tais comunidades de conhecimento, mas a cultura popular talvez esteja. (Jenkins, 2008 pg. 178)

Essa força do coletivo foi colocada em prática, em agosto de 2009 pelos fandoms do American Idol, em especial o do participante Adam Lambert, segundo colocado da oitava edição do programa. Misturando marketing e ação social, o cantor, apoiado pela gravadora RCA lançou uma campanha, que ficou conhecida como "Guerra dos fandoms", cujo grupo de fãs que arrecadasse mais dinheiro para o Donors Choose ${ }^{6}$ ganharia um chat particular com ele.

\footnotetext{
${ }^{6}$ http://www.donorschoose.org Site filantrópico, cujo doador escolhe a Instituição beneficiada.
} 


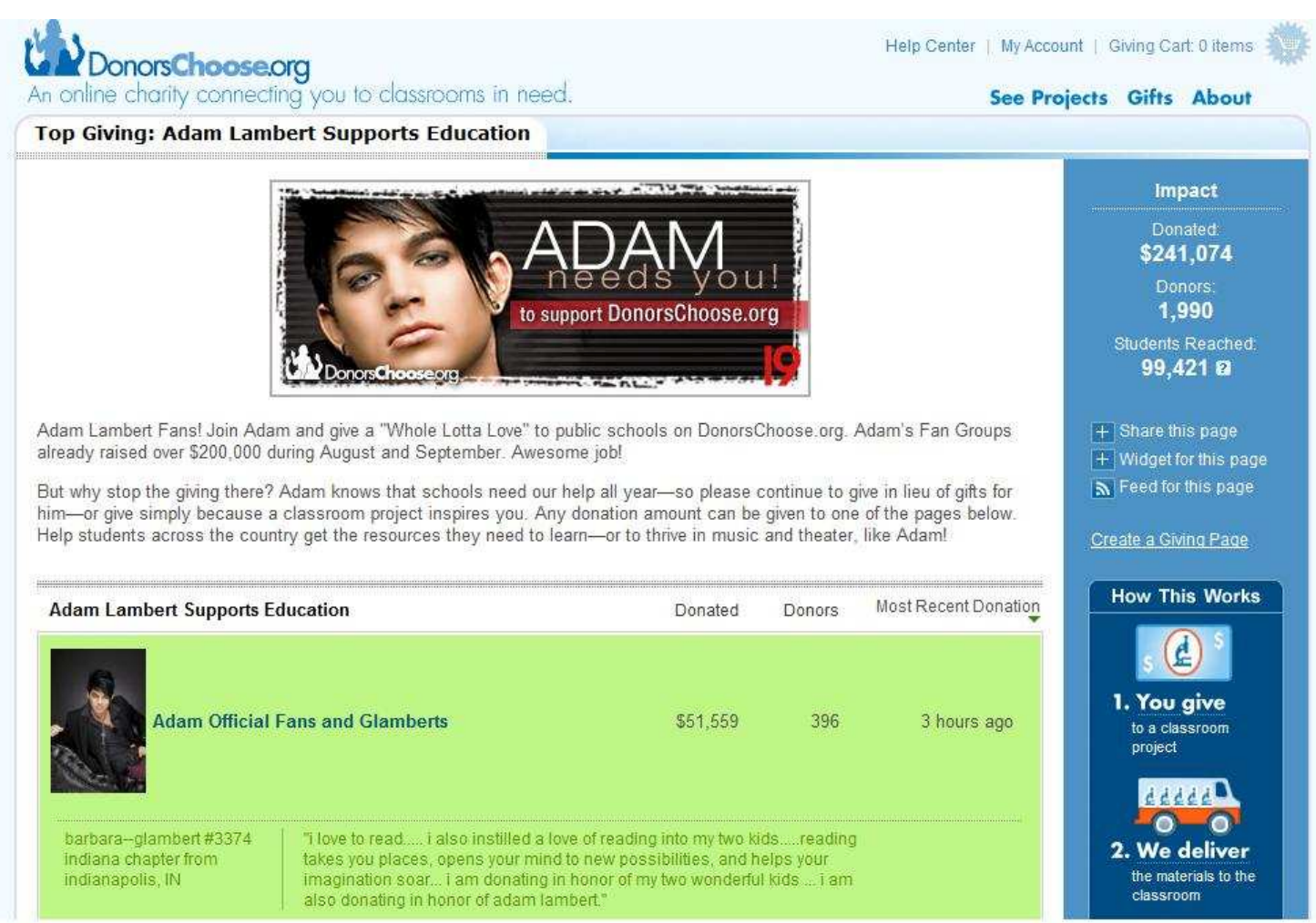

Fig 3: página de arrecadações do cantor Adam Lambert

Nada é mais importante para um grupo de fãs, do que contato "direto" com seu objeto de desejo, pois na grande maioria das vezes a única interação que ocorre entre fãs e ídolo é a parassocial (Rojek, 2005), ou seja, a construção de relações íntimas através da mídia ao invés de encontros presenciais. Por isso, a campanha teve um êxito fenomenal. Em três meses, os fandoms arrecadaram cerca de 241 mil dólares, e o grupo vencedor foi a comunidade ONTD_AI, cuja arrecadação chegou a 89 mil dólares. É interessante ver que as doações continuam até hoje, oito meses após o término da promoção. O que antes era apenas uma ponte ao ídolo, acabou tornando-se costume dos membros desses fandoms.

Além de promover campanhas entre os grupos de fãs do programa, desde a sexta edição do American Idol em 2006, o criador da franquia, Simon Fuller, organiza o programa "Idol Gives Back". O show que dura cerca de duas horas e passa no canal FOX da TV americana, já arrecadou mais de 140 milhões de dólares em prol de diversas ações comunitárias.

A participação de várias celebridades no evento é crucial para o envolvimento do público, que ao ver seu ídolo no programa, acaba participando de forma mais efetiva da causa. Desde presidentes até atores hollywoodianos e bandas consagradas, o Idol Gives 
Back (ou IGB, como denominado nos fóruns), além de reunir as chamadas celebridades classe A, leva até os países ajudados os ex-participantes do programa, que realizam shows e distribuem materiais arrecadados para os povos em questão.

A estratégia de arrecadação este ano foi baseada amplamente nas fanbases online dos ex e atuais idols que o programa lança. Em uma ação aonde as pessoas inscreviam-se no site IGB em diferentes times, cada um com o nome de um ex-idol, o grupo que obtivesse o maior número de arrecadações teria uma menção honrosa na edição ao vivo do programa.

Essa forma de ciberativismo (Vegh, 2008) através de uma intensa mobilização online entre as pessoas está sendo bastante incorporada pelos fãs da franquia American Idol. Fãs do ONTD e do fórum IDF montam equipes, criam sites, campanhas, e mutirões para doações. E essa ajuda não se restringe apenas ao Idol Gives Back, mas a uma série de programas sociais que os grupos comprometem-se em ajudar. O ONTD_AI inclusive possui uma página especial, o ONTD_AI gives back ${ }^{7}$, com o lema: “using our power for good".

\section{Remediação dentro dos fandoms}

No livro "Understanding new media" (1964: 29), Marshall McLuhan afirma que, "the content of any medium is always another medium. The content of writing is speech, just as the written word is the content of print, and print is the content of telegraph". Atualmente, a representação de uma mídia em outra, é denominada remediação (Bolter \& Gruisin, 1999).

Essa remediação é muito presente dentro dos fandoms, em forma de fanfics (histórias fictícias sobre os participantes do programa), fanarts (desenhos, gifs, macros dos participantes), fanzines (revistas feitas por fãs) e streams (transmissão do programa via internet.)

O grupo ONTD_AI além de possuir páginas específicas para fanfics, separadas por ordem alfabética, personagens e autores, é conhecido pelos gifs e macros que os usuários produzem. Muitos vídeos tirados do site oficial do programa e youtube são transformados em gifs, enquanto as fotos viram macros com frases cômicas, que fazem sucesso na web.

\footnotetext{
${ }^{7}$ http://community.livejournal.com/ontd_ai_gives.
} 
Uma das formas de remediação que faz bastante sucesso nos fandoms estudados são os streams ao vivo durante os programas. Sites como Rickey.org ${ }^{8}$, são especializados no assunto, e transmitem todos os anos os episódios, possibilitando que os fãs internacionais da franquia assistam o American Idol em tempo real.

Para Jenkins (2006: 135), "new tools and technologies enable consumers to archive, annotate, appropriate, and recirculate media content".

Com ajuda da tecnologia, a criação e construção dos fãs em cima do que já existe comprovam o fato de que nada é esgotado em uma única obra, nem em uma única mídia.

As conversas entre os fãs são expandidas em diversas direções, e são maiores que a própria franquia Idol. Seja criando grupos de discussão, montando linhas do tempo, revendo o mesmo vídeo dezenas de vezes para transcrever diálogos, especulando sobre o próximo programa, sobre os cantores e lançamentos de álbum. Os fãs estão sempre dispostos a buscar informações, a escavar até encontrarem o que desejam.

\section{Participação e convergência das mídias}

Discussões na web, sobre um programa de televisão cujo objetivo é encontrar um ídolo para a indústria fonográfica. Essa é a realidade transmidiática do American Idol. A participação dos grupos de fãs cada vez mais ganha voz perante os grandes conglomerados, que precisam dar atenção a essas culturas de nicho, que são extremamente dedicadas ao programa e lhe fazem manter seu status.

A participação é vista como uma parte normal da operação de mídia, e os debates atuais giram em torno das condições dessa participação. Assim como o estudo da cultura dos fãs nos ajudou a compreender as inovações que ocorrem às margens da indústria midiática, podemos também interpretar as estruturas das comunidades de fãs como a indicação de um novo modo de pensar sobre a cidadania e colaboração. (Henry Jenkins, 2008, p. 314)

A convergência dessas diversas mídias não precisa de uma distribuição específica, os conteúdos ali presentes passam por diversos canais de comunicação com múltiplos modos de acesso aos conteúdos, não possuindo um fluxo definido. Podendo ser descendente; dos grandes conglomerados para a população, ou então ascendentes; originados da própria cultura participativa.

\footnotetext{
${ }^{8}$ http://www.rickey.org. Site especializado em American Idol, e reality shows em geral.
} 
Os chamados gatekeepers, que originalmente são contra as produções provenientes da coletividade, estão tendo cada vez mais dificuldades em controlar os conteúdos culturais.

Enquanto os conglomerados que apostam no coletivo, dando ao consumidor a oportunidade de criar sua própria cultura ganham a confiança do público.

Dentro dos próprios fandoms, os participantes são estimulados a participarem cada vez mais, produzindo, pesquisando, criando novas formas de interação entre os usuários. Não basta saber, o importante é trocar informações, para que haja um crescimento da comunidade. Entre as características dos fóruns de mais sucesso do American Idol, é a criatividade e alta interatividade entre os membros que mais dão resultado.

Tanto a mídia alternativa, proveniente da cultura participativa, quanto a mídia vinda dos conglomerados, tem funções importantes na cultura em que vivemos. A primeira apresenta-nos ao novo e diversifica, a segunda tem o objetivo de expandir, divulgar, atingir todos os públicos possíveis. Unir as duas formas de mídia é a melhor maneira de obter-se sucesso no mercado atual. O American Idol é o exemplo da junção das duas mídias: fandoms, funcionando como mídia alternativa, apropriam-se dos conteúdos oferecidos pelo programa, que por sua vez, tem um alcance enorme em todo país e até fora dele, expandindo a franquia, dando material a população, que recria, modifica, conserta e devolve aos conglomerados algo diferenciado, e que muitas vezes é levado em consideração e modificado, formando assim um ciclo que nunca acaba.

\section{?. Conclusão}

Muito mais do que simples adolescentes gritando sem parar atrás de seu ídolo, os fãs são de extrema importância para a cultura participativa. Vítimas de preconceito e marginalização, os fandoms saíram do underground e pararam no mainstream, graças a força de seus participantes.

Engajados, criativos, e organizados, os fãs da franquia American Idol são autores, jornalistas, investigadores, fotógrafos, desenhistas, designers, e às vezes são todas essas profissões juntas. Tudo isso por um motivo maior: agregar valor a comunidade da qual fazem parte. 
A valorização de cada membro de um fandom é de suma importância para o crescimento do grupo, principalmente para que todos se sintam incluídos dentro da comunidade.

A apropriação de mídias existentes como ferramenta de comunicação e divulgação entre os interagentes, para futuramente serem reutilizadas e reinventadas, são características próprias dos fandoms.

Os grupos de fãs do ONTD_AI utilizam, por exemplo, o microblog twitter ${ }^{9}$, como ferramenta de divulgação de lançamentos de álbuns e singles de seus ídolos. Criando contas-spam, os usuários têm como principal objetivo, fazer com que as tags ${ }^{10}$ desejadas, apareçam nos "trending topics" publicidade gratuita.

Enfim, este trabalho procurou mostrar a importância dos grupos de fãs nos dias de hoje e a respectiva relação desses grupos alternativos, com os grandes conglomerados, retratando a importância da participação coletiva, e da influência cada vez maior desses grupos que antes eram marginalizados, e atualmente movimentam a indústria midiática.

\section{Referências Bibliográficas}

ANDERSON, Chris. A cauda longa. Rio de Janeiro: Elsevier Editora Ltda, 2006

BAYM, Nancy. "Talking about soaps: Communicative practices in a computermediated fan culture" pg: 111-130 em "Theorizing Fandom: fans, subcultures and identity". Nova Jersey: Hampton Press, 1998

FISKE, John. "The cultural Economy of fandom” pg 30-49 em "Adoring Audience: fan culture and popular media”. Nova Iorque: Routledge, Chapman and Hall, 1992

\footnotetext{
9 http://twitter.com. É uma rede social de microblogging aonde os usuários podem escrever até 140 caracteres..

${ }^{10}$ Tag é uma palavra-chave associada com uma informação que a descreve e permite uma classificação da informação baseada em seu significado.

${ }^{11}$ Tags mais comentadas no twitter que aparecem na página principal do microblog.
} 
BOLTER, David \& GRUISIN, Richard. Remediation: understanding new media. Massachusetts, Cambridge: MIT Press, 1999

JENKINS, Henry. Cultura da convergência. São Paulo: Aleph, 2008

Fans, bloggers and gamers: exploring participatory culture. Nova Iorque:

New York University Press, 2006

. Textual Poachers: television fans and participatory culture. Nova Iorque:

Routledge, Chapman and Hall, 1992

LEVY, Pierre. A inteligência coletiva: por uma antropologia do espaço. São Paulo: Edições Loyola, 1998

McLUHAN, Marshall. Os meios de comunicação como extensão do homem. São Paulo: Editora Cultrix, 1971

PRIMO, Alex. Interação mediada por computador: comunicação, cibercultura, cognição. Porto Alegre: Sulina, 2007

RECUERO, Raquel. Redes sociais na internet. Porto Alegre: Sulina, 2009

ROJEK, Chris. Celebridade. Rio de Janeiro: Rocco, 2001

ROBERTS, Kevin. Lovemarks: o futuro além das marcas. M. Books, 2005.

VEGH, Sandor. "Classifying Forms of online Activism - the case of cyberprotests against the world Bank" pg: 71-95 em "Cyberactivism: online activism in theory and practice”. Nova Iorque: Routledge, 2003. 
Reuista Anagrama: Reuista Científica Interdisciplinar da Graduação

Ano 4 - Edição 1 Setembro-nouembro de 2010

Auenida Professor Lúcio Martins Rodrigues, Ч४३, Cidade Uniuersitária, São Paulo, CEP: 05508-900

anagramagusp.br 